\title{
Comunidades de peces en desembocaduras de ríos y esteros de la Región de Valparaíso, Chile central
}

\author{
Fish communities in rivers mouths and streams of the Valparaiso Region, central Chile \\ Sergio Zunino ${ }^{1}$, Carolina Aliaga ${ }^{1}$ y Paola Da Venezia ${ }^{1}$ \\ ${ }^{1}$ Laboratorio Ecología y Biodiversidad, Departamento de Biología y Ciencias Ambientales, Facultad de Ciencias, \\ Universidad de Valparaíso, Gran Bretaña 1111, Playa Ancha, Valparaíso, Chile \\ sergio.zunino@uv.cl
}

\begin{abstract}
The fish community in 11 rivers mouths and estuaries in the Region of Valparaiso, was studied between 1999 and 2006. The fish collection was carried out through electro fishing. The conservation status and origin of species was determined. The results showed the presence of 23 species representing 10 orders, 14 families and 18 genera. Fourteen species $(68.8 \%)$ were found to be native and/or endemic, of which, 12 have conservation problems; Basilichthys microlepidotus is considered endangered, nine are vulnerable and two as inadequately known. In general, introduced species were the most abundant, including Cnesterodon decemmaculatus and Cheirodon interruptus which accounted for $62.7 \%$ of all fish collected, followed by the Chilean silverside with $15.6 \%$ while 20 other species had a low representation, as it happened with the endemic species Mordacia lapicida, Trichomycterus areolatus, Basilichthy australis and Percilia gillisi. This information will allow to recognize in a better way the effects of the activities to be developed in these ecosystems.
\end{abstract}

Key words: Biocenosis, freshwater fish

\section{Introducción}

La Región de Valparaíso es la región administrativa más pequeña del país, representando sólo el $0,8 \%$ de todo el territorio de Chile. Tiene un relieve bastante accidentado, donde los valles transversales (característicos del norte del país) son reemplazados por el valle longitudinal. En los ríos de la Región de Valparaíso dominan las hoyas originadas en la Cordillera de Los Andes, determinando dos rasgos hidrográficos interesantes. Uno, es el cambio de régimen de los ríos de pluvial a mixto (pluvionivoso), y el otro, es la estrechez relativa en el sentido latitudinal del área. Esto último genera cursos de agua de gran pendiente, como es el caso de los ríos, todos ellos con origen en la Cordillera de Los Andes (González et al. 1996), y que corresponden a los ríos Petorca, Ligua y Aconcagua que nacen en la región y un cuarto río, el Maipo, que se origina en otra región administrativa, pero
Resumen.- Se estudió la comunidad de peces de 11 desembocaduras de ríos y esteros de la Región de Valparaíso, entre 1999 y 2006 . La recolección de peces se realizó mediante electro pesca. Se determinó el estado de conservación y origen de las especies. Los resultados mostraron la presencia de 23 especies que representan a 10 órdenes, 14 familias y 18 géneros. Catorce especies $(68,8 \%)$ resultaron ser nativas y/o endémicas, de las cuales 12 presentan problemas de conservación; Basilichthys microlepidotus está considerada en peligro de extinción; nueve se encuentran en estado vulnerable y dos como inadecuadamente conocidas. En general, las especies más abundantes fueron las introducidas entre las cuales Cnesterodon decemmaculatus y Cheirodon interruptus correspondieron al $62,7 \%$ de todos los peces recolectados, seguidas por el pejerrey chileno Basilichthys microlepidotus, con 15,6\% del total, mientras las otras 20 especies tuvieron una baja representación, tal como sucedió con las endémicas Mordacia lapicida, Trichomycterus areolatus, Basilichthy australis y Percilia gillisi. Esta información permitirá reconocer con mayor facilidad los efectos de las actividades que se desarrollen en estos ecosistemas.

Palabras clave: Biocenosis, peces de agua dulce

desemboca en la de Valparaíso. Esta situación ha sido una de las principales causas de la escasa diversidad de peces, descrita por Eigenmann (1927). Por otra parte y a causa de la pronunciada inclinación de los ríos y esteros, los peces nativos son de pequeño tamaño y con escaso desarrollo locomotor; ello les ha permitido colonizar sólo hasta los 1.500 a $2.000 \mathrm{msnm}$ (Vila \& Pardo 2006). Por otra parte, hay hoyas hidrográficas menores que nacen en la Cordillera de la Costa, y que son de alimentación pluvial con algunos aportes de aguas subterráneas y que corresponden a los 19 esteros que desembocan al mar.

La riqueza de peces de agua dulce en Chile es un parámetro con una variación importante. Arratia (1981) señaló 52 especies, entre nativas y exóticas. Ruiz \& Marchant (2004) la extendieron a 75 especies. Vila et al. (2006) rebajaron esta cifra a 66 (44 especies nativas y endémicas). Froese \& Pauli (2008) reconocen la existencia de 67 especies de las cuales 49 son nativas y 
endémicas. A pesar de estas diferencias, el valor final siempre resulta extremadamente exiguo al compararlos con las 8.000 especies de peces de agua dulce de América del Sur y Central (Vari \& Malabarba 1998). Incluso, la riqueza nacional es igualmente muy pobre al compararla con las de otros ríos o esteros de Sudamérica. En tal sentido, Demonte \& Arias (2005) señalaron sólo para los ríos Paraná y Uruguay, una riqueza de 66 y 85 especies, respectivamente. Fuentes \& Rumiz (2008) informan de 124 especies para el río Paraguá de Bolivia. Rengifo (2007) describe para tres ríos de la región amazónica del Perú, una riqueza de 60 especies para el río Yurúa, 97 para el Breu y 92 para el Beu, o las 68 especies señaladas por Apone et al. (2008) para el río Quilombo de Sao Paulo, Brasil.

A pesar que los estudios sobre la fauna de peces continentales chilenos han tenido un fuerte incremento en las dos últimas décadas (Habit et al. 2006), aquellos relacionados con las desembocaduras de la Región de Valparaíso son aún escasos. Se cuentan con los aportes de Eigenmann (1927), Moreno \& Revuelta (1968), Dazarola (1972), Duarte et al. (1971), Quiroz (1999) y Habit \& Victoriano (2005), entre los más reconocidos.

La baja riqueza íctica de las aguas continentales nacionales tiene al menos tres orígenes fundamentales: 1) Es respuesta al particular relieve del territorio, 2) Es causa de las modificaciones y contaminación de las aguas por las diversas actividades humanas (Habit 2003, Campos et al. 1998, Habit et al. 2006, Vila et al. 2006, Habit et al. 2007). 3) Es resultado de la introducción de peces (Dazarola 1972, Welcomme 1988). En este último caso se manifiesta en aumentos de las distribuciones y abundancias de los peces introducidos, situación que ha provocado una fuerte merma en las poblaciones de peces nativos. A nivel nacional, esta disminución de la riqueza de especies nativas ya la mencionaban De Buen (1959) y Arratia (1978). Huaquín \& Manríquez (1986) han medido el efecto de los peces introducidos sobre los nativos. El ejemplo más claro lo señala Villwock (1992), para las especies de perca multicolor del género Haplochromis del Lago Victoria en África Oriental, cuya abundancia y riqueza han disminuido dramáticamente después de la introducción de la perca del Nilo - Lates niloticus (Linnaeus), señalando que numerosas especies endémicas están amenazadas o bien, ya han desaparecido por causa de la introducción de esta especie.

Las relaciones que se establecen entre especies nativas e introducidas dependen de numerosas acciones negativas simultáneas que se establecen entre ellas, entre las cuales destacan los tamaños corporales relativos de las especies en conflicto. De acuerdo a este fenómeno, Rincón et al.
(2002) han reconocido que Gambusia holbrooki (Girard) ha provocado la declinación de al menos dos especies de peces en España - Aphanius iberus (Valenciennes) y Valencia hispanica (Valenciennes) - en conjunto a importantes cambios ambientales, como destrucción y degradación de hábitats, eutrofización, extracción y contaminación de las aguas y alteraciones de los flujos, entre otros. En el mismo sentido, Mills et al. (2004) consideran a Gambusia holbrooki como predador agresivo que consumen una amplia variedad de organismos acuáticos incluso sus propios juveniles, reduciendo a las poblaciones nativas hasta su extinción, como ha sucedido con Iotichthys phlegethontis (Cope), en ambientes cerrados. Macchi et al. (2006) demuestran, bajo condiciones controladas, que los salmónidos son más eficientes en predar sobre Galaxias maculatus (Jenyns) que Percichthys trucha (Valenciennes).

Considerando que la biodiversidad de peces dulceacuícolas de Chile es poco diversa y que se encuentra afectada en grado desconocido, por la agresividad de los peces introducidos (De Buen 1959, Dazarola 1972, Arratia 1978, Welcomme 1988, Vila et al. 1999), este trabajo pretende establecer la composición y estructura de las comunidades de peces en 11 desembocaduras de la Región de Valparaíso, con énfasis en la relación entre las especies nativas o endémicas con las introducidas.

\section{Material y métodos}

Se analizaron $11(47,8 \%)$ desembocaduras de ríos y esteros exorreicos de la Región de Valparaíso, comprendiendo en total, 49 días de muestreos, desde 1999 a 2006 (Fig. 1, Tabla 1). Se consideró como desembocadura a toda el área del cuerpo de agua desde la orilla de mar hasta $5 \mathrm{~km}$ tierra adentro.

La recolección de peces se realizó mediante electro pesca utilizando para ello un generador eléctrico portátil Plus de $220 \mathrm{~V}$ con adaptador de voltaje. La pesca se efectuó recorriendo 100 metros de orilla. En aquellos sectores que presentaban orillas particularmente vegetadas la captura se efectuó con chinguillos de mano de malla de $5 \mathrm{~mm}$ de apertura. Los peces capturados luego de ser identificados y cuantificados fueron liberados en el mismo punto de recolección y una muestra pequeña se depositó en la colección de peces de agua continentales del Museo de Historia Natural de Valparaíso. A cada especie de pez, se les consideraron sus atributos de estado de conservación y origen de acuerdo a Campos et al. (1998) y Vila \& Pardo (2006).

Las recolecciones fueron complementadas con revisiones de las colecciones de peces de agua dulce pertenecientes al Museo Nacional de Historia Natural, 

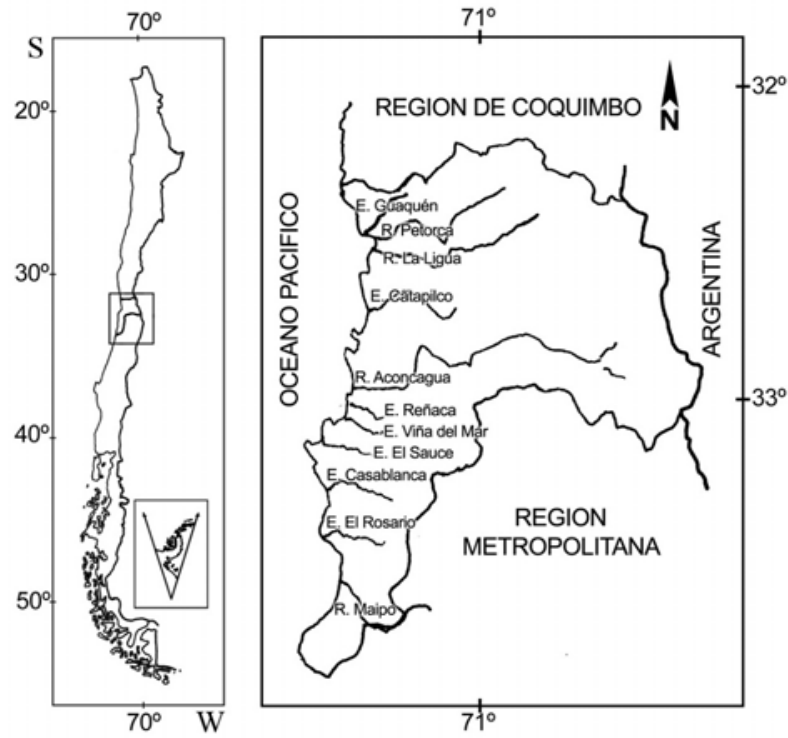

Figura 1

Mapa de la Región de Valparaíso mostrando los sitios estudiados

Map of Valparaiso Region showing the studied sites

Museo de Historia Natural de Valparaíso, Pontificia Universidad Católica de Valparaíso y Universidad del Mar.

Para cada desembocadura se estimó la riqueza de especies (S), la diversidad general con el índice de Shannon-Weaner $\left({ }^{+} \mathrm{H}\right)$ y de la diversidad máxima, ambas en $\log$ base 10; la similitud taxonómica fue estimada mediante el índice de Jaccard, todos ellos calculados mediante el programa Biodiversity (1997, versión 2).

\section{Resultados}

Se contabilizaron 4.339 peces, pertenecientes a 23 especies que representan a 10 órdenes, 14 familias y 18 géneros. El orden Perciformes fue el mejor representado con cinco familias; hay cinco órdenes con una sola familia, todas monotípicas. La familia Atherinidae fue la más diversa, con dos géneros y seis especies (Tabla 2).

Las especies más abundantes en las desembocaduras estudiadas fueron las introducidas, entre las cuales Cnesterodon decemmaculatus (Jenyns) y Cheirodon interruptus (Jenyns) correspondieron al 62,7\% de todos los peces recolectados, seguido por Basilichthys microlepidotus (Jenyns) con 15,6 \%, mientras que las otras 20 especies mostraron una baja representación (Tabla 2).

De las 23 especies de peces identificadas para los cuerpos de agua considerados, 14 (68,8\%) especies nativas (10 spp.) y/o endémicas (4 spp.). De este total, 12 especies presentan alguna consideración de conservación, resaltando B. microlepidotus que es considerada en peligro de extinción (Glade 1993, Campos et al. 1998, Habit \& Victoriano 2005); nueve se encuentran en estado de vulnerables y dos como inadecuadamente conocidas Mordacia lapicida (Gray) y Odontesthes mauleanum (Steindachner). Las especies nativas y/o endémicas son las que manifiestan con mayor claridad una relación entre bajas presencias en los diferentes cuerpos de agua y abundancias escasas, salvo B. microlepidotus, especie presente en ocho esteros o ríos (Tabla 2). Las otras nueve especies son introducidas y se encontraron en todas las desembocaduras consideradas y al mismo tiempo presentaron abundancias poblacionales mayores, como ocurrió con C. decemmaculatus y

Tabla 1

Ríos y esteros analizados, coordenadas UTM y fechas de muestreo

River and streams analyzed, geographic coordinates and sampling dates

\begin{tabular}{llll}
\hline \multicolumn{1}{c}{ Cuerpo de agua } & \multicolumn{2}{c}{ Coordenadas } & \multicolumn{1}{c}{ Fechas de muestreo } \\
\hline Estero Guaquén & 6439551 & 284991 & $10-15 / 10 / 1999,11-12 / 03 / 2006$ \\
Río Petorca & 6432958 & 287960 & $16-19 / 01 / 2003$ \\
Río Ligua & 6429690 & 290857 & $30 / 01-4 / 02 / 2003$ \\
Estero Catapilco & 6418523 & 287322 & $11 / 04 / 2002,6 / 05 / 2002$ \\
Río Aconcagua & 6401789 & 282975 & $5 / 10 / 2003,29 / 05 / 2004,27 / 12 / 2004$ \\
Estero Reñaca & 6392897 & 282229 & $7 / 04 / 2005,11 / 12 / 2005$ \\
Estero Viña del Mar & 6345171 & 281406 & $27-30 / 12 / 1999,18 / 06 / 2005,14 / 04 / 2006,11 / 11 / 2006$ \\
Estero El Sauce & 6339500 & 275927 & $18-20 / 07 / 1999,10-12 / 12 / 1999$ \\
Estero Casablanca & 6328431 & 277113 & $24-27 / 11 / 2000,3 / 12 / 2000$ \\
Estero del Rosario & 6317319 & 276435 & $2 / 03 / 2003,18 / 06 / 2004$ \\
Río Maipo & 6312883 & 276537 & $25-27 / 01 / 2002,28 / 06 / 2003$ \\
\hline
\end{tabular}




\section{Tabla 2}

Estado de conservación ( $P=$ en peligro de extinción, $V=$ =vulnerable, $I=$ =inadecuadamente conocida). Origen ( $E=$ endémica, $N=$ nativa, $\mathrm{I}=$ introducida). Abundancia de peces en las desembocaduras estudiadas y frecuencia y abundancia total, número total de ejemplares. Diversidad, $\mathrm{H}^{+}$y diversidad máxima $\mathrm{H}^{+}{ }_{\max }$ Las letras identifican a autores que han señalado la presencia de la especie en la desembocadura estudiada. a=Eigenmann (1927), b=Moreno \& Revuelta (1968), c=Duarte et al. (1971), d=Neira (1984), e=Dazarola (1972), $f=$ Quiroz (1999), g=González et al. (1996), h=Brito (2002)

Conservation status ( $\mathrm{P}=$ on danger of extinction, $\mathrm{V}=$ =vulnerable, $\mathrm{I}=$ inadequately known). Origin ( $\mathrm{E}=$ =endemic, $\mathrm{N}=$ =native, $\mathrm{I}=$ introduced). Fish abundance in the rivers mouths studied and total frequency and abundance, total number of specimens. Diversity $\mathrm{H}^{+}$and maximun diversity $\mathrm{H}^{+}{ }_{\text {max. }}$. Letters identify authors that have reported the presence of the species in the rivers mouths studied. a=Eigenmann (1927), b=Moreno \& Revuelta (1968), c=Duarte et al. (1971), d=Neira (1984), e=Dazarola (1972),

f=Quiroz (1999), g=González et al. (1996), h=Brito (2002)

\begin{tabular}{|c|c|c|c|c|c|c|c|c|c|c|c|c|}
\hline 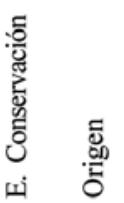 & 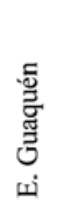 & $\begin{array}{l}\frac{J}{2} \\
\frac{0}{0} \\
\stackrel{0}{0 .}\end{array}$ & 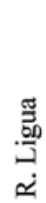 & త్ & 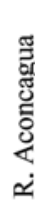 & 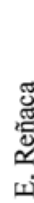 & 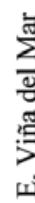 & $\frac{\mathscr{U}}{\tilde{J}}$ & & 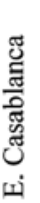 & 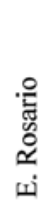 & \\
\hline
\end{tabular}

\begin{tabular}{|c|c|c|c|c|c|c|c|c|c|c|c|c|c|c|c|c|}
\hline \\
\hline Mordacia lapicida & I & $\mathrm{E}$ & - & - & - & - & d & - & - & - & - & - & - & - & 1 & - \\
\hline \multicolumn{17}{|l|}{ Characidae } \\
\hline Cheirodon interruptus & & I & - & 1 & - & 88 & 220 & - & $310 \mathrm{f}$ & 99 & 182 & - & 10 & 910 & 7 & 20,90 \\
\hline Cheirodon pisciculus & $\mathrm{V}$ & $\mathrm{N}$ & - & - & 1 & - & 1 & - & $2 \mathrm{f}$ & - & - & - & 1c & 5 & 4 & 0,11 \\
\hline \multicolumn{17}{|l|}{ Trichomycteridae } \\
\hline Trichomycterus areolatus & $\mathrm{V}$ & $\mathrm{E}$ & - & - & 59 & 4 & 1 & - & $6 \mathrm{f}$ & - & 23 & - & $4 \mathrm{c}$ & 97 & 6 & 2,23 \\
\hline \multicolumn{17}{|l|}{ Galaxiidae } \\
\hline Galaxias maculatus & $\mathrm{V}$ & $\mathrm{N}$ & - & - & - & $\mathrm{E}$ & 5 & - & - & - & 1 & - & $1 \mathrm{c}$ & 7 & 4 & 0,16 \\
\hline \multicolumn{17}{|l|}{ Poeciliidae } \\
\hline Cnesterodon decemmaculatus & & I & - & 7 & 80 & - & 24 & $1 \mathrm{~b}$ & $367 \mathrm{~b}$ & 1211 & 14 & 40 & $49 \mathrm{~b}$ & 1793 & 9 & 41,3 \\
\hline Gambusia holbrooki & & I & 14 & 1 & 124 & 114 & 2 & - & $4 \mathrm{f}$ & 26 & 1 & 65 & c & 350 & 10 & 8,07 \\
\hline \multicolumn{17}{|l|}{ Atherinidae } \\
\hline Basilichthys australis & $\mathrm{V}$ & $\mathrm{E}$ & - & - & - & - & a & - & - & - & - & - & - & - & 1 & \\
\hline Basilichthys microlepidotus & $P$ & $\mathrm{~N}$ & - & 91 & 134 & 38 & $60 \mathrm{a}$ & - & $98 \mathrm{e}$ & 7 & 244 & - & 7 & 679 & 8 & 15,60 \\
\hline Odontesthes brevianalis & $\mathrm{V}$ & $\mathrm{N}$ & - & - & - & - & - & - & - & - & - & - & c & & 1 & \\
\hline Odontesthes mauleanum & I & $\mathrm{N}$ & 31 & - & - & - & - & - & - & - & - & - & 8 & 39 & 2 & 0,90 \\
\hline Odontesthes regia & & $\mathrm{N}$ & - & $\mathrm{g}$ & g & $63 \mathrm{e}$ & $\mathrm{e}$ & - & - & - & 5 & - & - & 68 & 5 & 1,57 \\
\hline Odontesthes bonariensis & & I & - & - & - & - & - & - & $8 \mathrm{f}$ & - & - & - & - & 8 & 1 & 0,18 \\
\hline \multicolumn{17}{|l|}{ Perciliidae } \\
\hline Percilia gillissi & V & $\mathrm{E}$ & - & - & - & - & - & - & - & - & - & - & c & - & 1 & \\
\hline \multicolumn{17}{|l|}{ Percichthyidae } \\
\hline Percichthys trucha & $\mathrm{V}$ & $\mathrm{N}$ & - & - & - & - & - & - & - & - & - & - & $\mathrm{c}$ & - & 1 & - \\
\hline \multicolumn{17}{|l|}{ Mugilidae } \\
\hline Mugil cephalus & $\mathrm{V}$ & $\mathrm{N}$ & 1 & 2 & 20 & $\mathrm{E}$ & $50 \mathrm{e}$ & 67 & $2 \mathrm{f}$ & - & 19 & 1 & $18 \mathrm{c}$ & 180 & 10 & 4,15 \\
\hline \multicolumn{17}{|l|}{ Eleginopsidae } \\
\hline Eleginops maclovinus & $\mathrm{V}$ & $\mathrm{N}$ & - & - & - & - & - & - & - & - & - & - & c & - & 1 & - \\
\hline \multicolumn{17}{|l|}{ Cichlidae } \\
\hline Cichlasoma facetum & & I & - & - & - & 1 & 1 & - & $15 \mathrm{f}$ & - & 16 & - & - & 33 & 4 & 0,76 \\
\hline \multicolumn{17}{|l|}{ Cyprinidae } \\
\hline Carassius carassius & & I & - & - & 24 & - & - & - & - & - & - & - & $3 \mathrm{c}$ & 27 & 2 & 0,62 \\
\hline Cyprinus carpio & & I & - & - & - & E & 1 & - & $112 \mathrm{f}$ & - & 15 & - & $9 \mathrm{c}$ & 137 & 5 & 3,16 \\
\hline Tinca tinca & & I & - & - & - & - & - & - & - & - & - & - & c & - & 1 & - \\
\hline \multicolumn{17}{|l|}{ Acipenseridae } \\
\hline Acipenser transmontanus & & I & - & - & - & - & - & - & - & - & - & - & $4 \mathrm{~h}$ & 4 & 1 & 0,09 \\
\hline \multicolumn{17}{|l|}{ Clupeidae } \\
\hline Strangomera bentincki & & $\mathrm{N}$ & - & - & 2 & - & - & - & - & - & - & - & - & 2 & 1 & 0,04 \\
\hline TOTAL & & & 46 & 102 & 444 & 308 & 365 & 68 & 924 & 1343 & 519 & 106 & 114 & 4339 & & \\
\hline DIVERSIDAD $\mathrm{H}^{+}$ & & & 0,309 & 0,219 & 0,713 & 0,623 & 0,544 & 0,033 & 0,616 & 0,169 & 0,589 & 0,309 & 0,898 & & & \\
\hline DIV. MÁXIMA H Máx. & & & 0,477 & 0,788 & 0,95 & 0,954 & 1,11 & 0,301 & 1,00 & 0,602 & 0,100 & 0,477 & 1,26 & & & \\
\hline
\end{tabular}


Gambusia holbrooki con una abundancia del 41,3\% y $8,07 \%$, respectivamente. Por otra parte, las cuatro especies endémicas ( $M$. lapicida, Trichomycterus areolatus (Valenciennes), Basilichthys australis (Eigenmann) y Percilia gillisi Girard) estuvieron muy pobremente representadas salvo $T$. areolatus que estuvo presente en seis cuerpos de agua aunque con una abundancia muy baja $(2,2 \%)$. En el caso de las especies nativas, nueve de las diez presentaron riquezas más bien bajas al igual que sus abundancias, la excepción fue $B$. microlepidotus que estuvo presente en ocho desembocaduras con un 15,6\% de abundancia, que fue el valor más alto de presencia entre todas las especies tanto nativas como endémicas (Tabla 2). Diez especies estuvieron representadas por un solo ejemplar.

La desembocadura del río Maipo fue la más rica, con 17 especies, y el estero Reñaca, el más pobre, con sólo dos especies. Ambas desembocaduras poseen las diversidades generales y máximas mayores y menores, respectivamente. Con respecto a la riqueza de especies en los ríos y esteros, se determinó la formación de agrupamientos de los cuerpos de agua. Un primer grupo, de alta diversidad y coincidente con los ríos de mayor caudal - Maipo y Aconcagua. Un segundo grupo, compuesto por tres esteros - Casablanca, Viña del Mar y Catapilco - y por el río Ligua, se manifiestan con una riqueza específica muy semejante, de 9 a 10 especies. Finalmente, un tercer grupo, de riqueza baja y con valores muchos más heterogéneos, que oscilan entre seis especies para el río Petorca y dos especies para el estero Reñaca (Fig. 2, Tabla 3).

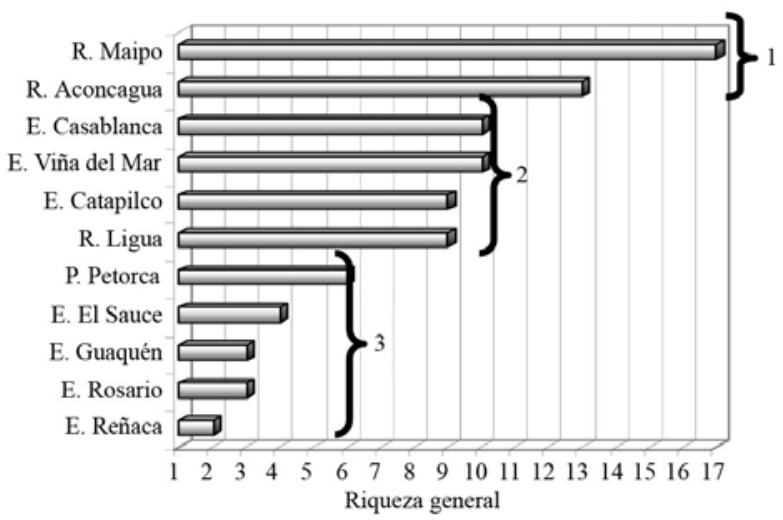

Figura 2

Riqueza de especies para cada una de las desembocaduras. 1 a 3=grupos de esteros y ríos

Species richness at each river mouth. 1 to $3=$ streams and rivers groups

El agrupamiento de las desembocaduras, de acuerdo al índice de Jaccard, permitió reconocer la existencia de un par de esteros, Casablanca y Catapilco, que poseen el mayor valor $\left(S_{J}=90 \%\right)$, puesto que comparten nueve especies y se diferencian porque Cnesterodon decemmaculatus sólo está presente en el estero Casablanca. A este grupo se encuentra asociado el río Aconcagua, con un valor menor (77\%), que comparte con ellos las nueves especies pero difiere por presentar cuatro especies exclusivas, de las cuales una sólo está en este río (B. australis). A este conjunto se asocian, con el mismo valor de similitud (66\%), los esteros de Viña del Mar, El

Tabla 3

Riqueza de especies de peces nativas e introducidas en los esteros y ríos estudiados

Species richness of native and introduced fish in the studied streams and rivers

\begin{tabular}{lcccc}
\hline & Total especies & Spp. introducidas & Sp. nativas & Spp. Introd/Spp. nat \\
\hline E. Guaquén & 3 & 1 & 2 & 0,50 \\
R. Ligua & 9 & 3 & 6 & 0,50 \\
R. Aconcagua & 13 & 5 & 8 & 0,64 \\
R. Maipo & 17 & 7 & 10 & 0,70 \\
E. Catapilco & 9 & 4 & 5 & 0,80 \\
R. Petorca & 6 & 3 & 3 & 1,00 \\
E. Reñaca & 2 & 1 & 1 & 1,00 \\
E. Casablanca & 10 & 5 & 5 & 1,00 \\
E. Viña del Mar & 10 & 6 & 4 & 1,50 \\
E. Rosario & 3 & 2 & 1 & 2,00 \\
E. El Sauce & 4 & 3 & 1 & 3,00 \\
\hline
\end{tabular}




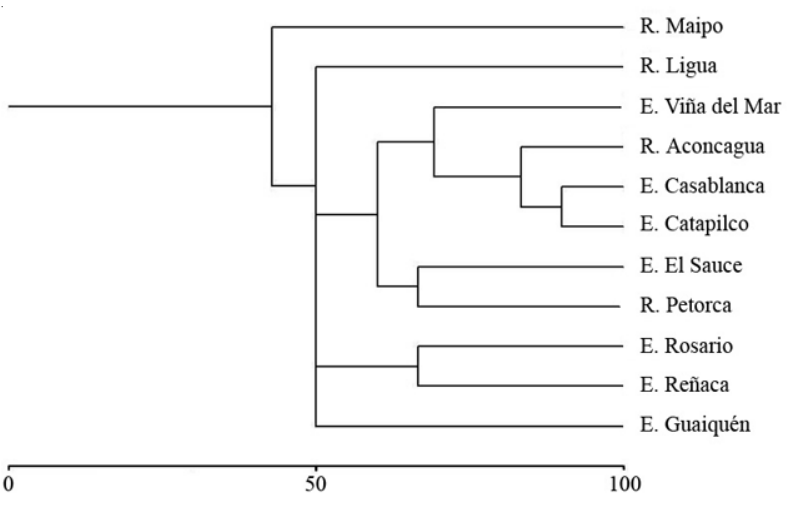

Figura 3

\section{Dendrograma de similitud taxonómica} según índice de Jaccard

Taxonomic similarity dendrogram according to Jaccard index

Sauce y el río Petorca, grupo al que se unen las desembocaduras de los esteros Rosario y Reñaca. Los esteros Guaquén y río Ligua son dos corrientes de agua que se conectan con el grupo anterior, al 50\% de similitud. El río Maipo es el único cuerpo de agua separado del conjunto de ríos y esteros, aunque su separación es muy pequeña, a consecuencia de ser el río de mayor riqueza de especies ( $\mathrm{S}=17$ ) (Fig. 3, Tabla 2).

De acuerdo a la riqueza de especies introducidas vs. nativas y endémicas, se identifican cuerpos de agua en donde priman las especies nativas y endémicas (esteros Guaquén y Catapilco y ríos Ligua, Aconcagua y Maipo); en otros, las exóticas son las más numerosas (esteros Viña del Mar, Rosario y El Sauce) o, por último, la relación se mantiene constante con un valor de 1 , como se reconoce para el río Petorca y los esteros Reñaca y Casablanca (Tabla 3).

\section{Discusión y conclusiones}

Del total de especies presentes $(n=23)$, en las 11 desembocaduras, se reconoce que tres especies dan cuenta del $78 \%$ de la abundancia total. De éstas, dos son introducidas (Cnesterodon decemmaculatus y Cheirodon interruptus) las que se encuentran en nueve y siete cuerpos de agua, respectivamente. La otra es el pejerrey de escama Basilichthys microlepidotus, especie nativa y presente en ocho desembocaduras. A pesar de su alta presencia (15,6\%), dicho valor podría reducirse por diversas causas, entre ellas sería producto de la fragmentación de los ecosistemas acuáticos, y probablemente uno de los más importantes sea la fuerte disminución de los caudales de los ríos por extracción exagerada de sus aguas como lo ha mencionado Vila et al. (2006). Esta última situación se debe a que los ríos ubicados en las zonas desérticas del país (entre las Regiones de Tarapacá hasta la Metropolitana) las demandas acuáticas superan los caudales disponibles; e incluso en períodos de sequía, los sobrantes que llegan a las desembocaduras son prácticamente nulos, situación que se extiende hasta el río Rapel (3389’S-7150’W) (Matus et al. 2004).

Las especies Mordacia lapicida, Galaxias maculatus, Eleginops maclovinus (Valenciennes), Strangomera bentincki (Norman), Acipenser transmontanus Richardson, Odontesthes regia (Humboldt) y Mugil cephalus Linnaeus corresponden a peces diadrómicos o anfídromos de baja frecuencia salvo las dos últimas que poseen altas presencias, puesto que se encuentran en $5(45 \%)$ y $10(91,5 \%)$ de las desembocaduras, respectivamente (Tabla 2). Esta baja presencia podría ser un reflejo de la escasa riqueza natural de la ictiofauna nacional como respuestas, en toda la zona central de Chile, a las fuertes pendientes de los ríos y esteros así como por las fuertes irregularidades de sus caudales (Eigenmann 1927, Vila \& Pardo 2006, entre otros) y a las cuales deben sumarse los efectos de la contaminación y de la alteración de las corrientes de agua como ha sido señalado por Huaquín \& Manríquez (1986), Escobar (2002), Ruiz \& Marchant (2004) y Vila et al. (2006), entre otros. Probablemente, además de la contaminación acuática, el embancamiento de las desembocaduras sea uno de los factores de importancia que produciría efectos en la morfología costera y en los ecosistemas y recursos naturales y en donde los sedimentos sirven de ligandos orgánicos a muchos contaminantes (Escobar 2002), o constituyen barreras físicas impidiendo el ingreso o salida de especies migratorias, considerando, según Habit \& Victoriano (2005), que el 71\% de las especies encontradas en los estuarios se presentan, igualmente en el océano y, que el 52\% se encuentran en ríos. Esto estaría limitando el intercambio de la fauna entre los ambientes dulceacuícolas y marinos.

Un manejo adecuado de las aguas servidas provoca una recuperación de la composición y/o estructura de la comunidad de peces de aguas dulce, como ha sido señalado por Quiroz (1999) para el estero de Viña del Mar, como producto de la puesta en marcha del gran colector de aguas servidas de Valparaíso, Viña del Mar, Quilpué y otras ciudades. Más recientemente, Habit et al. (2005) también demostraron que la actividad de una planta de tratamiento de aguas servidas ha permitido reconocer que al cabo de sólo cinco meses de funcionamiento, un sector del río Quilques (Los Ángeles, región central de Chile) muestra que la ictiofauna ha iniciado una recolonización del sistema acuático.

Para la situación chilena, el impacto de las especies introducidas sobre las nativas se reconoce como una de 
las mayores amenazas y que ha sido denunciada hace bastante tiempo por diversos autores nacionales, tales como De Buen (195), Campos (1970), Arratia (1978) entre otros; situación que se ve agravada por la falta de información en muchas especies chilenas (Habit et al. 2006a). Según Habit et al. (2006b), las únicas especies que han aumentado su distribución, en la última década, en el río Bíobío, son aquellas que soportan muy bien la degradación de los ecosistemas acuáticos, como ocurre con las especies introducidas: Gambusia holbrooki y Cyprinus carpio Linnaeus.

En términos generales, la fauna de peces nativos o endémicos en las desembocaduras no presenta un patrón de exclusividad, aunque se puede reconocer que aquellas desembocaduras con caudales más constantes y grandes, como son los ríos Ligua, Aconcagua y Maipo y los esteros Catapilco, Viña del Mar y Casablanca, presentan las mayores riquezas específicas.

Las especies nativas, a pesar de estar presentes en todas las desembocaduras, tienen una representatividad bastante baja e incluso son superadas por las introducidas como sucede en el río Petorca y los esteros Reñaca, Viña del Mar, El Sauce y El Rosario. De las especies nativas, Mugil cephalus es la más frecuente, ya que se encuentra en 10 cuerpos de agua y está ausente en el estero El Sauce, cuya barra sólo se abre en invierno, período en que podrían entrar ejemplares de esta especie y tal vez otras diadromas.

En el estero Guaquén se reconoce la presencia de Odontesthes mauleanum, especie endémica, que de acuerdo a su distribución geográfica según Dyer (2000) y a las características del hábitat preferido, no debiera encontrarse en dicha desembocadura, aunque Ruiz \& Marchant (2004) indican que se distribuye desde Valparaíso al sur.

Los actuales resultados permiten ampliar la información existente, en especial para los esteros Guaquén, Catapilco, Viña del Mar (mal citado como Marga-Marga), El Sauce, Casablanca y El Rosario. Por otra parte, llama la atención la baja riqueza del río Aconcagua ( $n=13$ spp.) con respecto al Maipo $(n=17$ spp.), lo cual nos permite hacer nuestras las proposiciones de Habit et al. (2006), ya que los peces de agua dulce son particularmente vulnerables a los cambios globales y han sido fuertemente impactados por la regulación y el uso de las aguas continentales. Por otra parte, las pérdidas o modificaciones de los hábitat acuáticos son responsables de las extinciones de especies nativas. En las últimas décadas, contaminaciones por descargas excesivas de nutrientes han provocado importantes cambios de los ecosistemas (Whitfield \& Elliott 2002, Lévêque et al. 2008, Habit et al. 2006).
Las especies de peces dulceacuícolas introducidas en Chile están afectando a las especies nativas o endémicas de manera casi desconocida, aunque se reconoce que las especies introducidas, al ser de mayor talla y/o más móviles que las nativas pueden provocar reducciones poblacionales significativas al predar sobre los individuos adultos (Mills et al. 2004); o si las especies introducidas son de pequeño tamaño, como es el caso de Gambusia holbrooki, éstas predan selectivamente sobre los alevines o los huevos de especies endémicas o nativas de mayor tamaño.

\section{Agradecimientos}

Al Dr. Bryan Dyer por todo su apoyo en la identificación de peces. Al Dr. Luis Malabarba de la Pontificia Universidad Católica Do Rio Grande Do Sul, por su gran ayuda en la identificación de los representantes del género Cheirodon en la región de Valparaíso. Al Dr. Hernán Gaete por su continuo apoyo en la confección del manuscrito, a mi gran amigo, José Cáceres quien hizo la revisión final del manuscrito y a los evaluadores de la revista por las sabias sugerencias que han contribuido a mejorar el manuscrito.

\section{Literatura citada}

Apone F, AK de Oliveira \& JC Garavello. 2008. Composicao da ictiofauna do río Quilombo, tributario do río MogiGuacu, bacia do alto río Paraná, sudeste do Brasil. Biota Neotropica 8(1): 93-107.

Arratia G. 1978. Comentarios sobre la introducción de peces exóticos en aguas continentales de Chile. Ciencias Forestales 1(2): 21-30.

Arratia G. 1981. Géneros de peces de aguas continentales de Chile. Publicación Ocasional, Museo Nacional de Historia Natural 34: 1-108.

Brito J. 2002. Primer registro de Acipenser transmontanus Richardson, 1863, asilvestrado en la desembocadura del Río Maipo, San Antonio, Chile Central. Noticiario Mensual, Museo Nacional de Historia Natural 347: 9-11.

Campos H. 1970. Introducción de especies exóticas y su relación con peces de agua dulce de Chile. Noticiario Mensual, Museo Nacional de Historia Natural 162: 3-9.

Campos H, G Dazarola, B Dyer, L Fuentes, JF Gavilán, L Huaquín, G Martínez, R Meléndez, G Pequeño, F Ponce, VH Ruiz, W Sielfeld, D Soto, R Vegas \& I Vila. 1998. Categorías de conservación de peces nativos de aguas continentales de Chile. Boletín, Museo Nacional Historia Natural 47: 101-122.

Dazarola G. 1972. Contribution a l'étude de la faune ichthyologique de la région Valparaíso Aconcagua (Chili). Annales de Limnologie 8(1): 87-100.

De Buen F. 1959. Los peces exóticos en las aguas dulces de Chile. Investigaciones Zoológicas Chilenas 5: 103-135. 
Demonte L D \& JD Arias. 2005. Ictiofauna de afluentes de los ríos Paraná y Uruguay en la Provincia de Entre Ríos, Argentina. Insugeo Misceláneas 14 :355-366.

Duarte W, R Feito, C Jara, C Moreno \& E Orellana. 1971. Ictiofauna del sistema hidrográfico del Río Maipo. Boletín, Museo Nacional de Historia Natural, Chile 32: 227-268.

Dyer B. 2000. Revisión sistemática de los pejerreyes de Chile (Teleostei, Atheriniformes). Estudios Oceanológicos 19: 99127.

Eigenmann C. 1927. The fresh water fishes of Chile. Memoirs of the National Academy of Sciences 22: 1-63.

Escobar J. 2002. La contaminación de los ríos y sus efectos en las áreas costeras y el mar. CEPAL. Serie Recursos Naturales e Infraestructura 50: 1-68.

Froese R \& D Pauli (eds). 2008. FishBase. World Wide Web electronic publication.[disponible en] <www.fishbase.org > [07/2008].

Fuentes V \& DI Rumiz. 2008. Estudio preliminar de la ictiofauna y los hábitats acuáticos del Río Bajo Paraguá, Santa Cruz, Bolivia. Biota Neotropica 8(1): 74-81.

Glade A (ed). 1993. Libro Rojo de los Vertebrados Terrestres de Chile, 68 pp. CONAF, Santiago de Chile.

González A, T Gezan \& H Manríquez. 1996. Geografía de Chile, Geografía V Región de Valparaíso, 297 pp. Instituto Geográfico Militar, Santiago de Chile.

Habit E. 2003. Calidad del agua del estero Piduco (Talca, VII Región): Un análisis basado en la data existente. Teoría 12: 43-54.

Habit E \& P Victoriano. 2005. Peces de agua dulce de la Cordillera de la Costa. En: Smith-Ramírez C, J Armesto \& C Valdovinos (eds). Historia, biodiversidad y ecología de los bosques costeros de Chile, pp. 392-406. Editorial Universitaria, Santiago de Chile.

Habit E, O Parra \& C Valdovinos. 2005. Ictiofauna de un sistema fluvial receptor de aguas servidas: respuesta a una nueva planta de tratamiento (río Quilques, Chile Central). Gayana 69(1): 94-103.

Habit E, B Dyer \& I Vila. 2006a. Estado de conocimiento de los peces dulceacuícolas de Chile. Gayana 70(1): 100-113.

Habit E, MC Belk, RC Tuckfield \& O Parra. 2006b. Response of fish community to human-induced changes in the Biobio river in Chile. Freshwater Biology 51: 1-11.

Habit E, M Belk, P Victoriano \& E Jaque. 2007. Spatiotemporal distribution patterns and conservation of fish assemblages in a Chilean coastal river. Biodiversity and Conservation 16(11): 3179-3191.

Huaquín LG \& A Manríquez. 1986. Problemática y perspectivas de los peces nativos de aguas continentales chilenas. $2^{\circ}$ Encuentro Científico Medio Ambiente, Vol 1: 132-138. CIPMA, Talca.

Lévêque C, T Oberdorff, D Paugy, MLJ Stiassny \& PA Tedesco. 2008. Global diversity of fish (Pisces) in freshwater. Hydrobiologia 595(1): 545-567.
Macchi PJ, MA Pascual \& PH Vigliano. 2006. Differential piscivory of the native Percichthys trucha and exotic salmonids upon the native forage fish Galaxias maculatus in Patagonian Andean lakes. Limnologica 37: 76-87.

Matus N, B Fernández, M Prado \& S Larraín. 2004. Recursos hídricos de Chile: Desafíos para la sustentabilidad, 172 pp. LOM ediciones, Santiago de Chile.

Mills MD, RB Rader \& MC Belk. 2004. Complex interactions between native and invasive fish: the simultaneous effects of multiple negative interactions. Oecologia 141: 713-721.

Moreno C \& G Revuelta. 1968. Un nuevo pez en aguas continentales chilenas: Cnesterodon decemmaculatus (Jenyns, 1842). Noticiario Mensual, Museo Nacional de Historia Natural 12(143): 8-11.

Neira F. 1984. Biomorfología de las lampreas parásitas chilenas Geotria australis Gray, 1851 y Mordacia lapicida (Gray, 1851) (Petromyzoniformes). Gayana Zoología 48(1-2): 3-40.

Quiroz S. 1999. Ictiología de poblaciones de peces en el estero de Viña del Mar. Seminario de Título, Universidad de Playa Ancha de Ciencias de la Educación, Valparaíso, 71 pp.

Rengifo B. 2007. Diversidad de peces en la cuenca del Alto Yuruá (Ucayali, Perú). Revista Peruana de Biología 13(3): 195-202.

Rincón PA, AM Correas, F Morcillo, P Risueño \& J LobónCerviá. 2002. Interaction between the introduced eastern mosquitofish and two autochthonous Spanish toothcarps. Journal of Fish Biology 61: 1560-1585.

Ruiz V \& M Marchant. 2004. Ictiofauna de aguas continentales chilenas, 356 pp. Departamento de Zoología, Universidad de Concepción, Concepción.

Vari R \& L Malabarba. 1998. Neotropical ichthyology: An Overview. En: Malabarba LR, RE Reis, RP Vari, ZM Lucena \& CAS Lucena (eds). Phylogeny and classification of Neotropical fishes, pp. 1-11. EDIPUCRS, Porto Alegre.

Vila I, R Pardo, B Dyer \& E Habit. 2006. Peces límnicos: diversidad origen y estado de conservación. En: Vila I, A Veloso, R Schlatter \& C Ramírez (eds). Macrófitas y vertebrados de los ecosistemas límnicos de Chile, pp. 72102. Editorial Universitaria, Santiago de Chile.

Vila I, L Fuentes \& M Contreras. 1999. Peces límnicos. Boletín, Museo Nacional de Historia Natural 48: 61-75.

Vila I \& R Pardo. 2006. Peces límnicos. En: CONAMA(ed). Biodiversidad de Chile: Patrimonio y desafíos, pp. 306311. Ocho Libros Editores, Santiago de Chile.

Villwock W. 1992. Consecuencias de la introducción de peces exóticos sobre las especies nativas del lago Titicaca. Ecología en Bolivia 23: 49-56.

Welcomme RL. 1988. International introductions of inland aquatic species. FAO Fisheries Technical Paper 294: 1-318.

Whitfield AK \& M Elliott. 2002. Fishes as indicators of environmental and ecological changes within estuaries: a review of progress and some suggestions for the future. Journal of Fish Biology 61 (Suppl. A): 229-250. 